\title{
Gianni Celati, il cinema di John Cassavetes e la distruzione dei dispositivi
}

\section{Andrea Rondini}

\section{(2) OpenEdition}

1 Journals

Edizione digitale

URL: https://journals.openedition.org/cher/766

DOI: $10.4000 /$ cher.766

ISSN: 2803-5992

\section{Editore}

Presses universitaires de Strasbourg

\section{Edizione cartacea}

Data di pubblicazione: 9 juillet 2020

Paginazione: $175-187$

ISBN: 979-10-344-0068-3

ISSN: 1968-035X

Notizia bibliografica digitale

Andrea Rondini, «Gianni Celati, il cinema di John Cassavetes e la distruzione dei dispositivi», reCHERches [Online], 24 | 2020, online dal 20 septembre 2021, consultato il 17 novembre 2021. URL: http://journals.openedition.org/cher/766 ; DOI: https://doi.org/10.4000/cher.766 


\title{
Gianni Celati, il cinema di John Cassavetes e la distruzione dei dispositivi
}

\author{
ANDREA RONDINI ${ }^{1}$
}

$\mathrm{I}^{\mathrm{n}}$ ricchi e articolati rapporti tra Celati e il cinema (in un'accezione che comprende anche il documentario) si esplicano su diversi livelli: citazioni dirette o indirette di film, attori o registi nei testi narrativi (in Verso la foce addirittura nel medesimo paragrafo si trovano John Ford e Leopardi; Celati 2016c. 1024); numerosi interventi critici; realizzazione di pellicole di non fiction, progetti e collaborazioni (Palmieri 2016a: CIV). Il cinema diviene allora un indicatore sul quale misurare la propria poetica: «Si può fare in modo da produrre per iscritto l'effetto di una smorfia di Stan Laurel?» (Celati 2008a).

La critica stessa ha toccato questi temi fin dagli anni Settanta e ormai la vasta bibliografia su questo specifico aspetto del lavoro di Celati ha assunto, per quantità e rilevanza, una posizione importante negli studi sullo scrittore (tanto che risulterebbe incongruo citarne solo qualche specimen). Ci si occuperà qui del rapporto tra Celati e un importante regista americano, John Cassavetes, che tra la fine degli anni Sessanta e la prima metà dei Settanta firma alcune delle sue opere più rilevanti. L'interesse di Celati per Cassavetes si manifesta esplicitamente:

Nell'universo filmistico americano l'idea d'un puro accadere o d'un correre gratuito del tempo con cui si consuma naturalmente la vita di tutti, forse è un'idea intrattabile. Per cui non può esistere la nozione positiva di momenti vuoti che si riempiono di pensieri, in una divagazione senza meta. Perché nell'ideologia americana anche il pensare ha senso solo come un attivismo calcolato in base a un fine. Forse solo nei film di Cassavetes il cinema americano ha trovato figure apertamente incapaci di controllare la propria esistenza, e capaci di sprecarla andando a spasso e parlando e rimuginando a vanvera (Faces, Husbands, A Woman Under the Influence). (Celati 2011a: 137)

Sarà forse utile, per capire queste parole, delineare, almeno brevemente, le tappe della carriera di Cassavetes, il cui percorso da cineasta indipendente lo colloca in una posizione anomala rispetto al sistema americano mainstream

1 Andrea Rondini, Università di Macerata. 
e lo rende certamente interessante agli occhi di Celati, sempre polemico nei confronti dell'apparato hollywoodiano ${ }^{2}$ (anche se lo scrittore non tutto rigetta dell'industria cinematografica statunitense, soprattutto nella sua epoca classica; Iacoli 2011: 74-75). I due, tra l'altro, sono quasi coetanei: Cassavetes è nato nel 1929 (muore a soli 60 anni nel 1989), Celati nel 1937. Occorre notare, almeno en passant, che anche Pasolini, pensando a un cinema di poesia, aveva Cassavetes tra i suoi autori di riferimento (Pasolini 2004: 1580).

Dopo gli esordi negli anni Cinquanta in qualità di attore di alcuni serials televisivi e in film con registi importanti per la sua formazione come Martin Ritt (Edge of the City, 1957), Cassavetes fonda nel circuito off-Broadway l'Actor Workshop (operandovi in qualità di insegnante, produttore, regista): Shadows nascerà come saggio collettivo di recitazione e del resto partecipazione, improvvisazione/spontaneismo, identificazione totale con il ruolo sono elementi chiave della pratica cassavetesiana. I protagonisti di Shadows sono tre fratelli afroamericani, Lelia, Hugh, Ben, questi ultimi musicisti jazz, anche se il solo Hugh effettivamente tenta - con scarsi risultati - di entrare nel circuito professionistico. Si tratta di personaggi introversi, insoddisfatti e inconcludenti, talvolta in grado di trasformarsi in irriverenti perditempo metropolitani (come durante la visita di Ben e dei suoi amici allo Sculpture Garden del Moma). Ma soprattutto la differenza di Cassavetes rispetto al sistema comunicativo hollywoodiano si vede nella predilezione verso personaggi ambigui e chiaroscurali. Si consideri in proposito il tema del posare e dell'atteggiarsi dei tre fratelli; essi recitano una parte, portano una maschera, sapendo di portarla e sapendo altresì quanto gli sia utile per difendersi dalle ferite della vita: vi è quindi una coscienza serio-comica - che è pure fondamentale struttura espressiva e stilistica ibrida - che si muove tra compiacimento e dolore (Carney 1994: 50-51).

A Shadows seguono Too Late Blues (1961), focalizzato sul tormentato rapporto con il successo di un giovane jazzista che abbandona il suo gruppo di musicisti marginali e outsiders, e A Child Is Waiting (1963) in cui si conferma l'interesse per un mondo di diversi ed emarginati (qui bambini portatori di handicap). D'ora in avanti il regista alternerà la personale produzione filmica, innovativa e sperimentale, con la partecipazione come attore a produzioni di un certo spessore ma comunque destinate al mercato, fondamentali per finanziare i propri progetti.

Già da questo micro-ritratto affiorano alcune omologie con il mondo celatiano: la concezione dell'artista come outsider; la predilezione per marginali, solitari e 'pascolanti'; le componenti comiche; l'improvvisazione come metodo di recitazione ma anche come occasione di spreco vitale, dissipatorio (emblematica l'estemporanea partita a baseball di Too Late Blues), cui sarà da aggiungere la tendenziale dissoluzione della trama come usuale struttura diegetica. Il jazz stesso

2 «[S]crivendo o leggendo dei racconti si vedono paesaggi, si vedono figure, si sentono voci: è un cinema naturale della mente, e dopo non c’è più bisogno di andare a vedere i film di Hollywood» (Celati 2016e: 1271). 
si configura come vettore di relazione tra i due artisti, visto che Celati guarderà sempre a quella grammatica musicale come enzima di ricodifica liberatoria della lingua allo stesso modo in cui i personaggi eccentrici rappresentano la potenziale liberazione dalla realtà convenzionale. Il jazz è funzionale tra l'altro, nell'autore che letterariamente ne incarna lo spirito, Céline, a costruire un personaggio che teatralizza il suo corpo (Celati 2008b: 109). Il Celati del 1971 quindi colloca l'autore francese nel perimetro della recitazione (affine allo stesso Cassavetes che, come si notava sopra e si vedrà ancora, costruisce film in cui il personaggio vive mettendosi in scena).

Le riflessioni sul regista prima riportate non si configurano quindi come casuali - seppur profonde - notazioni bensì si collegano a questioni centrali della poetica di Celati e vengono infatti poste in relazione con una serie di significative componenti del suo laboratorio, Hemingway e Bob Dylan ${ }^{3}$, sul quale lo scrittore si soffermava già nel suo corso al DAMS di Bologna nell'autunno del 1973 (Palmieri 2016a: XCVI). Il puro accadere è visibile proprio nel Krebs di Hemingway, uno dei solitari americani studiati da Celati (Celati 2006a: 38); del resto, tutta l'umanità ritratta da Cassavetes potrebbe appartenere a questa categoria, visto che la loro vita perde la ricchezza dell'esperienza e scompare nell'anomia, le due dorsali concettuali che certificano lo status di solitario ${ }^{4}$.

Si tratta in effetti di elementi che innervano tutta la riflessione di Celati, intrecciandosi sia sul piano critico che su quello creativo: considerando la già citata "divagazione senza meta», si vedano i riferimenti alla prosa leopardiana, erratica e frammentaria (Celati 2016a: 243), alla sua dimensione filosofica «senza mete prefissate» (ibidem), alla sua natura dissipatoria (ivi: 244-245); e similmente alla scrittura di Delfini, errabonda e «senza nessuna focalizzazione prospettica» (ivi: 259) e ancora a quella di Manganelli e di Cavazzoni (Rivelazioni sui purgatori presenta un «andamento senza meta»; ivi: 272). Ma, su tutti, occorre considerare Ariosto con i suoi eroi che "girano a vuoto" (ivi: 51-53 e passim). La vita come accadimento casuale e serie di momenti vuoti, l'azione non finalizzata, il tempo sospeso, il girovagare per itinerari imprecisi erano peraltro esemplarmente raffigurati, sub specie cinematografica, dalla Strada (1954) di Fellini ${ }^{5}$, titolocronotopo che prefigura tra l'altro una delle spazialità cassavetesiane tipiche.

3 «L'idea di una vita gratuita, del vivere per vivere, è poco accettabile nel modo di pensare americano medio. Ma si ritrova nei racconti di Hemingway e d'altri autori, come nelle canzoni dei nostri folk-singers, fino a quelle di Bob Dylan» (Celati 2008c: 137). Sull'incidenza dell'idea e della pratica del canto in Celati si veda Della Rovere 2019.

4 Si veda per esempio la presentazione del personaggio femminile di uno dei racconti di Three lives (1909) di Gertrude Stein: «La figura di questa ragazza sempre pronta a mescolarsi con gli altri, e sempre separata da tutti, mostra una solitudine non più riducibile alla trasparenza del senso comune. Nell'irrisolta esistenza di Melanchta, lo smarrimento e la normalità, l'isolamento individuale e la socialità, sembrano diventati la stessa cosa, la stessa spersonalizzazione nell'anonimato di massa» (Celati 2006a: 33).

5 Cfr. Celati 2008b: 136: «Qui non c'è nessuna azione finalizzata. Tutto è sospeso nel 
A sua volta, la narrativa di Celati si focalizza più volte sull'antiparadigma dello sprecare la vita andando a spasso. Si possono considerare, citando volutamente da un'opera del 'secondo' Celati come Cinema naturale: girare a caso senza saper dove andare (Celati 2016e: 1280), andare a spasso e girovagare come un accattone (ivi: 1388$)^{6}$, vagare in macchina di qua e di là (ivi: 1397), andare a vanvera (ivi: 1399); anche al Pucci dei Costumi degli italiani «L'unica cosa che gli piaceva era andare in giro tutto il giorno per le strade a caso» (Celati 2016f: 1660); l'azione non finalizzata scandisce pure Avventure in Africa, critica serrata alla patologica coercizione occidentale a imprimere sempre al tempo un obiettivo necessario e un percorso rettilineo nonché alla volontà di tenere tutto sotto controllo (Celati 2016d: 1226, 1138). Si tratta di una costellazione semantica ancorata al perimetro dell'erranza ${ }^{7}$, del vagare/errare che ibrida sia il vagabondaggio (come spostamento fisico-spaziale) sia il rifiuto - motore primo della poetica di Celati - dei significati fissi e prestabiliti della vita e delle parole, accettando invece la paura di 'sbagliare' e di lasciarsi prendere al laccio da fole e illusioni.

Ognuno dei film specificatamente menzionati da Celati rende perspicua questa rete di rapporti e ne arricchisce la prospettiva. Con Faces Cassavetes inizia quella erosione-distruzione del comportamento e dell'essere umano, del quale mette in luce tutto il rimosso psichico e l'animus premoderno, il ribollire di desideri, miserie ed errori, che caratterizzerà tutto il suo cinema. Come per Celati, si potrebbe dire che l'umanità cassavetesiana, pur nella sua variante nevrotica contemporanea e con le sue maschere che sono l'unica modalità con cui oramai si può contrattualizzare socialmente la propria alterità, si ispira a uno slancio pulsionale incontrollato, rabelaisiano e swifitiano ${ }^{8}$, antico. La celatiana fabulazione sarà il suo lessico (Celati 2001: 36-39).

Faces non ha una vera e propria trama, più che altro si configura come il pedinamento - in alcuni tratti semidocumentaristico - dei protagonisti: Richard e Maria, matura coppia in crisi, la prostituta Jeannie e i suoi clienti (tra cui Richard), il gigolo Chet che ha un'occasionale avventura con Maria. La

vago accadere in cui si consuma la vita degli uomini. Gelsomina, Zampanò, il Matto, sono figure di un'epoca in cui è finito ogni eroismo - figure di una vita di pura sussistenza, che non hanno nessun potere né un luogo dove abitare. Ma sono figure più che mai votate agli incontri che si riempiono di pensieri - quegli incontri su itinerari imprecisi e frammentari, nei momenti vuoti in cui niente ci attira, e dove ci si ritrova con altri in un tempo sospeso».

6 Si noti tra l'altro il possibile riferimento ad Accattone di Pasolini.

7 Sul recupero in questa prospettiva della Vita nova di Dante cfr. Camilletti 2016.

8 La Favola della botte spiega «i grandi fenomeni storici come spostamenti di impulsi biologici sublimati, di tipo escretorio e sessuale» ed equipara le "manifestazioni elette dello spirito a condizione paranoica. Nell'uno e nell'altro caso assistiamo ad una depravazione di modelli ideali di sublimazione dell'uomo occidentale, per mezzo della loro degradazione al rango di formazioni rimosse o represse della nostra cultura»; Celati 2006b; cfr. anche Milani 2019: 165-178. 
pellicola mostra anche formalmente una carica spiazzante e anticonvenzionale nella decisione di iniziare ex abrupto, nel non presentare i personaggi e nel non fornire allo spettatore informazioni circa le situazioni in cui si trovano: nelle scene d'apertura, Richard partecipa a un «unexplained meeting at an unspecified location for an unknown purpose» (Carney 1994: 79). Il film presenta inoltre una natura serio-comica: rapporti sentimentali usurati, tradimenti, uomini che sprecano il tempo e sembrano aver dismesso ogni direzione della loro vita per dedicarsi a un divertimento incostante e infantile, nonché irritante nella sua inconcludente ripetitività; le serate di Jeannie, trascorse in un claustrofobico appartamento, che dovrebbero essere per tutti appaganti, sono un incubo di tristezze e disperazione sorretto da una gioia forzosa e di facciata. Eppure, anzi proprio per questo, per ovviare a un vuoto e un odio di sé (anche esplicitamente espresso da Maria) il film pullula di risate: si può dire che il rapporto uomo-donna (sia esso di coppia o mercenario) si risolve, ancor più che nella relazione erotica, in una serie di risate, frizzi, lazzi, conditi di improvvise litigate, incomprensioni, riappacificazioni, vale a dire in sequenze di eccessi stonati (concetto, quello di eccesso, che potrebbe essere un ulteriore tassello per una lettura incrociata tra Cassavetes e Fellini, autore anch'egli votato alla distruzione del dispositivouomo con il suo cinema connotato da una poetica bachtiniana dell'eccesso e votato alla rappresentazione dell'umano come organismo ancestralmente desiderante; Celati 2009a e 2009b). In pieno '68 (vi è anche un fugace accenno al Vietnam) i personaggi di Faces giocano così al gioco di un triste e maldestro divertimento, l'imperativo della ormai realizzata società capitalista, che però qui gira a vuoto e male. Insomma: smile!, come non a caso si intitola una sezione dei solitari americani (Celati 2006a: 35-37).

Celati può probabilmente essere stato attratto dagli elementi di critica sociale, ma il film di Cassavetes mette in scena personaggi in preda all'erranza del desiderio, all'assenza di direzione esistenziale e che sprecano la vita. Gli uomini di Faces «are sprung free from the codes of decorum and normative expression that regulate behavior in most life and art. [...] characters give uninhibited expression to their wildest desires and most extreme impulses» (Carney 1994: 82-83). Tra l'altro queste tipologie comportamentali sono psichicamente borderline, alle soglie della follia (Carney 1994: 83). Chet rappresenta anch'egli questa tipologia umana, ma ha una importante differenza rispetto agli altri perché possiede l'esatta consapevolezza, la coscienza, della pantomima che tutti stanno interpretando (quasi come lo straniero di Teorema di Pasolini che invade e sovverte la casa altoborghese). La sua fuga sul tetto della villa, il suo guizzare via dopo che è stato sorpreso da Richard, il salto per raggiungere la strada, sono inoltre un inserto quasi da comica, e tutta la sezione ha i connotati dell'avventura (conoscenza, rapporto d'amore, tentato suicidio di Maria, istrionismi dopo il salvataggio, fuga). Inoltre la fuga di Chet si configura come riemersione di un motivo keatoniano, quello dell'esclusione («Keaton is often thrown out of a place»: West 2000: 21), utilizzato da Celati nella sua interpretazione di Beckett: «L'altro tema keatoniano [...] è quello dell'espulsione. Molto spesso Keaton 
viene espulso o buttato fuori da vari posti, ma soprattutto da una casa» (Celati 2001b: 184) ${ }^{9}$. Un destino condiviso da altri personaggi di Cassavetes, come il Moskovitz di Minnie e Moskovitz; peraltro un salto sigilla il finale de La banda dei sospiri.

Faces presenta poi un tessuto sonoro-fonico che ibrida e destabilizza la parola, immergendola polifonicamente in una serie di pre/post espressività vocali: «Il discorso, la risata, la tosse, il canto diventano oggetti sonori a pieno titolo» (Jousse 1997: 64), accompagnati da «rumori, risate, pianti, borborigmi, onomatopee, allitterazioni, giochi di parole, canzoni, balbettamenti, ripetizioni» (ivi: 65); dominante di questo universo sonoro è la debordante presenza del riso e della risata ${ }^{10}$, che accompagna la - fallimentare - esistenza dei personaggi con la sua iterata, drammatica e scomposta presenza. Sulla scrittura come abitata dalla sonorità-musicalità, Celati insiste in tutta la sua riflessione, dalle pagine su Cèline a quelle su Joyce a quelle più specificatamente narrative. In particolare il gioco di parole, l'uso ludicamente distorto e distruttivo della lingua ricorre in tutti gli autori della biblioteca di Celati, dal Bloom dell'Ulysses joyciano ai Marx Brothers (paradigmatico Duck Soup, 1933). La singolare tessitura ludica di Faces esplicita così una nervatura carnevalesca, modulata polifonicamente, destinata a riapparire nelle pellicole successive di Cassavetes, ed omologa alla rilevanza di Bachtin nelle pagine teoriche di Celati. La polifonia di Faces è stata infatti messa in relazione con le posizioni bachtiniane: "Il "comico" di Cassavetes ha di queste ascendenze (Faces inizia con delle risate scomposte e incongrue). Non ha nulla a che fare con i codici della "commedia" così come è andata istituzionalizzandosi attraverso la storia dei generi cinematografici [...] Il suo "comico" affonda le radici nella tradizione rituale carnevalesca» (Arecco 1980: 44).

Il film inoltre è una rappresentazione del lavoro registico sul corpo, in particolare il viso (ma non solo), ripreso nelle sue smorfie e deformazioni: non a caso Gilles Deleuze, uno degli interlocutori più importanti del discorso cinematografico dello scrittore (Celati 2011b: 37) ${ }^{11}$, insiste su questo aspetto, mettendo in evidenza la centralità del corpo e la sua preminenza nel cinema di Cassavetes rispetto alla trama e alla narrazione: "Quando Cassavetes dice che i personaggi non devono derivare dalla storia o dall'intreccio, ma che la storia deve essere la secrezione dei personaggi riassume l'esigenza di un cinema dei corpi: il personaggio è ridotto ai propri atteggiamenti corporei e quel che deve risultarne è il gestus, vale a dire uno "spettacolo", una teatralizzazione o una

9 Corsivo di Celati. Lo scrittore fa esplicito riferimento al concetto di espulsione elaborato da Coursodon 1973.

10 «La parola viene colta nella sua fondamentale impurità, inseparabile dagli accenti più acuti, dalle inflessioni più sorprendenti. [...] Faces è forse innanzi tutto un'esperienza sonora immensa e avvolgente, l'immersione sconvolgente in una lingua ignota. Cascata di monologhi ininterrotti e continuamente spezzati, che mettono in gioco una parola multiforme e infinitamente frammentata» (Jousse 1997: 64-65).

11 La figura di Deleuze è rilevante anche per la riflessione di Celati sull'arte: cfr. Martelli 2019b: 15-39. 
drammatizzazione valida per ogni intreccio. Volti è costruito su atteggiamenti del corpo presentati come volti che arrivano alla smorfia, esprimono l'attesa, la stanchezza, la vertigine, la depressione» (Deleuze 2010: 213-214). Il corpo e il gesto - dispositivo che disarticola l'umano e ne mina la meccanica coerenza, frantumandola in una serie di atti aberranti - erano tra l'altro già al centro delle riflessioni di un critico molto apprezzato da Celati, André Bazin (Celati 2008: 140). Ma ancora di più sarà da sottolineare come la motricità corporea evidenzi la radicalizzazione della lezione di Buster Keaton da parte di Cassavetes:

c'est le rythme des corps qui fait le montage, qui donne sa logique au récit: longs vomissements, luttes paniquées, étreintes rapides. Tous ces gestes impossibiles à recomposer, sans mémoire, sans conscience, organisés dans la sensation de l'instant, qui deviennent, au-delà de toute volonté, de toute intention, les motifs principaux de la trame. Avec Cassavetes la perte de soi s'accomplit dans l'exercice du corps, dans la dépense physique revendiquée. Ce n'est plus seulement une autre perception de l'espace, comme dans la ligne de Keaton [...], c'est [...] une temporalité qui s'organise autrement, quand disparaît la ligne abstraite qui ordonne les souvenirs et les projets (Amiel 1994: 138).

Tutto questo lo si ritrova in Husbands (1970). Il film si focalizza sulle peregrinazioni di Gus, Archie, Harry, tre quarantenni, dopo il funerale dell'amico Stuart: "per tre giorni si trasformano in buffoni, in giullari e si abbandonano a quelle licenze che il loro status sociale non consente loro di osare nella vita normale» (Arecco 1980: 50). I tre carnevalizzano la loro vita e ne perdono in qualche modo il controllo: dormono in metropolitana, compiono ragazzate per strada, si fanno 'attori' di fronte ai semiscandalizzati passanti, improvvisano una partita a basket in una palestra deserta e si trasformano definitivamente in satiri anarchici e bambineschi. Poi entrano in un pub del tutto casualmente e si ubriacano; la sbornia si tramuta in rissa verbale contro una anziana avventrice e in una pantomima grottesca caratterizzata da reiterate situazioni scatologiche, turpiloquio, liti, riappacificazioni; Harry torna a casa e litiga con la moglie e con la suocera. Quindi il terzetto prende l'autobus: Gus si esibisce come una sorta di clown con il gag della sigaretta che gli scivola ripetutamente dalla mano e delle quattro dita in bocca. Infine la partenza per Londra: in un casinò di lusso, Gus e Harry giocano e tentano alcuni approcci con le donne, lo stesso fa Archie ma in modo assai più imbarazzante e volgare; trovano infine tre ragazze. Dopo quest'ultima avventura Archie e Gus tornano a casa, mentre Harry decide di rimanere.

Husbands è una summa del cinema di Cassavetes e presenta una fitta rete di temi celatiani. La pellicola è in primo luogo una celebrazione del personaggio errante nello spazio aperto (anzi nell'Aperto, la dimensione geografico-mentale priva di dispositivi e di strutture precostituite) ${ }^{12}$. Questo tratto consente di instaurare ancora una triangolazione concettuale tra Cassavetes, Celati e Deleuze. Afferma il filosofo in L'immagine-movimento:

12 Per una presentazione di questo aspetto sia concesso il rimando a Rondini 2013: 58-63. 
Il girovagare aveva trovato in America le condizioni formali e materiali per un rinnovamento. Il girovagare si fa per necessità, interna o esterna, per bisogno di fuga. Ma adesso perde l'aspetto iniziatico che aveva nel viaggio tedesco (ancora nei film di Wenders), e che conserva malgrado tutto nel viaggio beat (Easy rider di Dennis Hopper e Peter Fonda). È diventato un girovagare urbano e si è staccato dalla struttura attiva e affettiva che lo dirigeva, gli imprimeva delle direzioni, per quanto vaghe. [...] la cosa più chiara del moderno girovagare è che si compie in uno spazio qualsiasi, stazione di smistamento, deposito abbandonato, tessuto indifferenziato della città [...]. Come dice Cassavetes, si tratta di disfare lo spazio, quanto la storia, l'intreccio o l'azione (Deleuze 2016: 4881-4887).

Si stabilisce così un nesso tra erranza, datità corporea e spazialità, triangolazione concettuale fondamentale di Celati, qui funzionale all'erosione dell'azione finalizzata e della sua codifica diegetica, la trama. Cassavetes viene ancora inserito da Deleuze proprio nella linea degli autori che hanno ridefinito la spazialità tramite la rappresentazione di luoghi astratti e deserti, che il filosofo definisce sconnessi e svuotati e che minano ancora una volta la stessa possibilità di azione da parte del soggetto; all'interno di questa strategia espressiva «i personaggi si trovavano sempre meno in situazioni sensoriomotrici "motivanti", ma piuttosto in uno stato girovagante, di bighellonaggio o erranza che definiva delle situazioni ottiche e sonore pure. L'immagine-azione tendeva allora a frantumarsi, mentre i luoghi determinati svanivano, lasciando emergere spazi qualsiasi dove si sviluppavano gli affetti moderni di paura, di distacco, ma anche di freschezza, di velocità estrema e di interminabile attesa»; in tale linea si inserisce lo stesso neorealismo e la Scuola di New York (Lumet e appunto Cassavetes), tesa a «disfare lo spazio»; non a caso Cassavetes ritorna nelle pagine dedicate alla crisi dell'immagine-azione (insieme a Lumet e Altman) in cui Deleuze mostra la dissipazione della grammatica dell'azione.

Husbands presenta anche un concentrato di tratti carnevaleschi: il riso, la deformazione parodica, il corpo grottesco, la promozione del cesso come luogo di decisioni vitali (Arecco 1980: 49 sgg.), in cui rientrano la stessa fenomenologia del nomadismo e la pratica del gag; in tal modo il film si apparenta alle pagine celatiane dedicate a Bachtin e al gag (Celati 2001). La vita capovolta dei mariti americani si inscrive perfettamente nel perimetro del Carnevale bachtiniano (nonché nei miti libertari degli anni Settanta): «Le gerarchie sociali, le attribuzioni di valore agli oggetti e alle parti del corpo, la tematica del rapporto tra carne e spirito, tutto ciò sembra scoppiare nel riso carnevalesco, invertendo il senso, abbassando ciò che è alto ed elevando ciò che è basso» (Celati 2001: 56) ${ }^{13}$. A sua volta, il gioco della sigaretta si configura come gag-immagine di natura estrema: «I gags più virtuosistici sono quelli del rapporto con un oggetto o con una serie di oggetti» (Celati 2001: 179). Il gag, poi, è un ulteriore dispositivo di distruzione, in questo caso della linearità diegetica: «quando la produzione

13 Esemplificativo di questo paradigma un grottesco dialogo del film tra Archie, Gus ed Harry: «Avrò mangiato qualcosa di cattivo - Forse della merda - Si vede che non era fresca». 
di gags assume l'importanza di forma canonica dell'atto di narrare, il testo si conforma come costellazione di tracce discontinue, di improvvisi accessi, di deviazioni ed ironie non costruttive, e insomma come serialità di interpolazioni» (ivi: 170). Celati mostrerà una perdurante fedeltà al gag, anche quando perderà la sua carica carnevalesca per diventare segno di rassegnata anomia ${ }^{14}$.

Il capovolgimento e il paradosso innescato da questi eredi dei giganti buffoni, nella loro mistione di situazioni comiche e solitudini esistenziali, tra voglia di gioco, rifiuto degli schemi e disperazione, si configura inoltre come una 'Hilarotragoedia' (l'opera di Manganelli su cui si sofferma Celati 1998: 254), sintassi espressiva già presente in altri momenti del cinema di Cassavetes, come il finale di Shadows, con l'accettazione sorridente di un destino di scacco altrimenti insopportabile (vedi anche Arecco 1980: 41); in tal modo lo stile di Husbands si inscrive nella linea delle narrazioni serio-comiche inaugurate da Luciano (Celati 1998: 270) atte a scardinare la logica dei generi e in cui la depressione può dare allegria (Celati in Palmieri 2016b: 1734-1735). In fondo i maturi padri di famiglia di Husbands, sono anch'essi protagonisti di comiche nonché coevi dei Clowns di Fellini usciti nel 1971 (sui quali Celati 2011a: 170) senza contare che i personaggi di Cassavetes sono infantili nel senso positivo della polarità aurorale in Celati (quella vichiana). In questa prospettiva ci si può chiedere se Husbands non riproduca un immaginario cinematografico assai caro allo scrittore, vale a dire quello dei Marx Brothers; La farsa dei tre clandestini (Celati 1987), la riscrittura celatiana dell'universo cinematografico dei Marx, può infatti costituire un'esperienza che istituisce una implicita linea di continuità tra la bagarre scatenata da Groucho, Harpo e Chico e i loro eredi, tre eredi, degli anni Settanta, peraltro ormai entrati nel perimetro dei solitari, con le loro esistenze spaccate tra fuga dalla famiglia (la vicenda del marito che si allontana da casa è topos codificato dal Wakefield di Hawthorne) e ritorno (comunque solo per due di loro: Gus e Archie rientrano, almeno momentaneamente, nella normalità come il Flitcraft, del Falcone maltese di Hammett; Celati 2006a: 40).

Si può dire che il cinema di Cassavetes prediliga, come l'opera di Celati, situazioni e vite in bilico sull'abisso ${ }^{15}$. E infatti tali sono quelle di A Woman Under the Influence, catalizzate in Mabel, la protagonista del film. Mabel è la moglie di Nick Longhetti, ruvido capocantiere, dal quale ha avuto tre figli; figura lunatica ed eccentrica, sempre associata alla musica, la donna personifica il mondo alla rovescia e soprattutto, nella sua clownerie, la coscienza infelice degli antichi giganti buffoni, diventata nella modernità humour, tendenza dello spirito, corporeità aerea (a indicare «un volo ideale al di sopra di tutte le miserie della quotidianità. [...] La comicità corporea si spiritualizza, [...] diventa astratta, ideale, schizoide» Celati 2001a: 93). La donna porta con sé una duplicità allegro-melanconica, non lontana da un'altra forma di doppiezza hilarotragica,

14 Cfr.: «rileggendo questi appunti, mi prenderò forse per un mediocre comico che ripete all'infinito gli stessi gag con suo figlio»; Celati 2016g: 981.

15 Con riferimento a Belpoliti 2016. 
la «doppiezza del puer senex» (Celati 2011a: 170, con riferimento ancora a $I$ clowns di Fellini) ${ }^{16}$; del resto Mabel è una possibile incarnazione del paradigmaAlice, nel suo essere un'istanza di flussi e metamorfosi ${ }^{17}$.

Significativa la scena della festa organizzata per i figli, evidenti e affini interlocutori di quel folletto ${ }^{18}$ che è Mabel: la donna trasforma il divertimento pomeridiano in una sorta di bagarre, ballando sulle note della Morte del cigno e invitando i figli e i loro piccoli ospiti a travestirsi, a liberare la propria inventiva, finendo però col provocare la reazione scandalizzata del genitore dei bambini invitati, gettato in una situazione che sfugge al suo controllo e al normale ordine delle cose. La danza della protagonista, nuova apparizione del corpo comico nello spazio e del mimo comico (Celati 2001: 93), nuda vita sciamanica prelogica e vichiana, sottolinea inoltre la sua forte carica fisico-spontaneistica (Carney 1994: 165-166) ed è tra l'altro una personificazione del lavoro registico di Cassavetes: il sollecitare gli altri ad uscire da se stessi, costringerli a rivelarsi fuori dalle maschere sono esattamente le azioni che il regista elegge come obiettivo del proprio cinema, concepito non come spettacolo istituzionale bensì come laboratorio di conoscenza di sé e al contempo un oltrepassamento/denudamento delle convenzioni comunicative.

Soprattutto, la donna deve spesso difendersi dal controllo e dalle istanze normalizzatrici della Famiglia, sprovvista della sensibilità e degli strumenti epistemologici per accettarne l'alterità; Mabel è infatti un'ulteriore reincarnazione della figura dell'Espulso: sorvegliata e punita - Foucault docet - dovrà patire sei mesi di internamento psichiatrico. Si conferma così il nesso tra estroversione e malattia decretata dai rappresentanti dell'ordine', a partire dal marito Nick che infatti viene ritratto come incapace di disfarsi dall'ossessione del controllo del lavoro, dei colleghi e appunto della moglie (Carney 1994: 158), nuova incarnazione del fool soggetto a persecuzione sottolineato da Celati nel Cèline di Guignol's band (Celati 2001: 107).

Nella sua stralunata teatralità, nella sua azione distruttiva oppure per la necessità di difendersi dagli altri, la donna è in ogni caso sempre in scena: quello di Mabel è un parlato come spettacolo, un vero repertorio in cui voice e gesture - per riprendere i termini impiegati da Celati - danno vita a un vero e proprio acting (Celati 2017), fatto di smorfie, soliloqui, alterazioni autoironiche della voce, pose corporee in atto di sfida (non lontane dai «gesti magici del maledire» e dalla «rabbia dello stizzoso» tipici del mimo comico; Celati 2001: 93). Gli stessi personaggi celatiani, perseguitati e fuori posto, amano spettacolarizzarsi: come Guizzardi, essi «si mettono in scena come se fossero degli attori» (SchwarzLausten 2002: 111) oppure si pensano come fabulatori ammalianti (Raccis 2018).

16 Cfr. anche il riferimento al Puer Aeternus e al pensiero di Hillman in Chierici 2011: 53.

$17 \mathrm{Su}$ questi aspetti di Alice disambientata nonché sulla sua complessa regia di allestimento, cfr. Martelli 2019a: 197-212.

18 Cfr. Arecco 1980: 72. 
Con Mabel arriva al capolinea la storia dei solitari americani di Cassavetes (e si noti che non pochi solitari di Celati sono in realtà solitarie, cioè donne); dell'esperienza hanno celebrato la scomparsa ridendo (Faces), errando (Husbands), mettendosi in scena e rimuginando (A Woman Under the Influence). Mabel deve infatti spesso allestire un dialogo io-io, una stanza mentale-espressiva tutta per sé che le consenta di gestire le sconfitte che derivano dal contatto con gli altri (e sarebbe interessante sviluppare più di un parallelo con alcune solitarierimuginatrici) ${ }^{19}$. Rimuginare è l'attività residuale su cui si chiude l'antologia dei solitari (Celati 2006a: 42) e non casualmente è parola chiave del lessico celatiano, basti pensare al protagonista di Lunario del paradiso che rimugina sul modello dell'Amleto ma anche a prove narrative posteriori, per esempio in Notizie ai naviganti (Celati 2016e: 1396). Rimuginare in fondo è un gesto, dove per gesto si deve intendere, con Agamben, la non-azione, la in-azione che disattiva l'umano ${ }^{20}$. La solitaria è così portatrice di una decostruzione dei dispostivi sociali e di discorso stabiliti e dello stesso concetto di azione finalizzata, rivendicando uno spazio di gratuità che non è solo 'gioco' - e gioco non lo sarà ancora per molto, come proprio il cinema evidenzia ${ }^{21}$ - ma rappresenta un fenomeno erosivo più profondo, teso a destituire l'azione e il finalismo del mortale logos teleologico della modernità. Il Cinema stesso, in fondo, e i film di Cassavetes lo dimostrano, è un grandioso fenomeno di messa in crisi del Discorso.

\section{Bibliografia}

Agamben G., 2017, Karman, Torino, Bollati Boringhieri.

Amiel V., 1994, «Le corps en image (de Buster Keaton à John Cassavetes)», Esprit, Février, p. 135-146.

Arecco S., 1980, John Cassavetes, Firenze, La Nuova Italia.

Belpoliti M., 2016, Gianni Celati, la letteratura in bilico sull'abisso, in Celati 2016b, p. XI-LXXII.

Camilletti F., 2016, «Vitae nove per la modernità: stilnovismo ed erranza del desiderio in Delfini e Celati», The Italianist, 36, p. 1-17.

Carney R., 1994, The films of John Cassavetes. Pragmatism, Modernism, and the Movies, Cambridge, Cambridge University Press.

Celati G., 1976, «Il corpo comico nello spazio», il verri, 3, p. 22-32.

Celati G., 1987, La Farsa dei Tre Clandestini, Bologna, Baskerville.

19 Cfr. Celati 2006a: 30-31 (a proposito di Story of an hour di Kate Chopin).

20 Cfr. Agamben 2017: 138.

21 L'ultima reincarnazione del solitario si ritrova «nel personaggio del solitario e perverso e cogitabondo, il killer patologico di mille film, che rimugina nel suo antro [...] il solitario diventato "l'uomo nero" per spaventare i bambini, segno invertito di quell'inferno che è la vita americana» (Celati 2006a: 49). 
Celati G., 2001, Finzioni occidentali. Fabulazione, comicità e scrittura, Terza edizione riveduta, Torino, Einaudi.

Celati G., 2006a, «Introduzione», in Storie di solitari americani, a cura di G. Celati e D. Benati, Milano, Rizzoli, p. 7-56.

Celati G., 2006b, «Swift, profetico trattato sull'epoca moderna», Zibaldoni e altre meraviglie, 5 luglio, <www.zibaldoni.it>.

Celati G., [1971], 2008a, "Caro Calvino, non mi hai capito», La Repubblica, 3 ottobre, <www.larepubbica.it $>$.

Celati G., [1971], 2008b, «La scrittura come maschera», in Céline L.F., Colloqui con il professor $Y$, Torino, Einaudi, p. 105-110.

Celati G., 2009a, «Fellini e il maschio italiano/1», Zibaldoni e altre meraviglie, 29 maggio, <www.zibaldoni.it $>$.

Celati G., 2009b, «Fellini e il maschio italiano/2», Zibaldoni e altre meraviglie, 5 luglio, <www.zibaldoni.it>.

Celati G., 2011a, Conversazioni del vento volatore, Macerata, Quodlibet.

Celati G., 2011b, "Quando ho visto Nel corso del tempo», in Documentari imprevedibili come $i$ sogni, a cura di N. Palmieri, Roma, Fandango, p. 33-39.

Celati G., 2016a, Studi di affezione per amici e altri, Macerata, Quodlibet.

Celati G., 2016b, Romanzi, cronache e racconti, a cura di M. Belpoliti e N. Palmieri, Milano, Mondadori.

Celati G., 2016c, Verso la foce, in Celati 2016b, p. 987-1098.

Celati G., 2016d, Avventure in Africa, in Celati 2016b, p. 1099-1268.

Celati G., 2016e, Cinema naturale, in Celati 2016b, p. 1268-1474.

Celati G., 2016f, Costumi degli italiani 1, parzialmente in Celati 2016b, p. 16571692.

Celati G., 2016g, Quattro novelle sulle apparenze, in Celati 2016b, p. 981.

Celati G., [1968] 2017, "Parlato come spettacolo», in Celati G., Animazioni e incantamenti, con C. Gajani, a cura di N. Palmieri, Roma, L'Orma Editore, p. 292-293.

Chierici A.M., 2011, La scrittura terapeutica. Saggio su Gianni Celati, Bologna, Archetipolibri.

Coursodon J. P., 1973, Buster Keaton, Seghers, Paris.

Deleuze G., [1985] 2010, L'immagine-tempo, Milano, Ubulibri.

Deleuze G., [1983] 2016, L'immagine-movimento, Torino, Einaudi, formato ebook.

Della Rovere A., 2019, «Riscrittura è traduzione. La tradizione come canto e discanto in Gianni Celati», in Gianni Celati. Traduzione, tradizione, riscrittura, a cura di M. Ronchi Stefanati, Roma, Aracne, p. 95-107.

Iacoli G., 2011, La dignità di un mondo buffo. Intorno all'opera di Gianni Celati, Macerata, Quodlibet.

Martelli M., 2019a, "Come Alice, attraverso Alice ovvero "tutto il quotidiano riscritto e rivisitato"», in Gianni Celati. Traduzione, tradizione, riscrittura, a cura di M. Ronchi Stefanati, Roma, Aracne, p. 197-212. 
Martelli M., 2019b, L’impensé du regard. Trois études sur Gianni Celati et les arts visuels, Macerata, Quodlibet.

Milani F., 2019, "La Favola della botte e il "felice vanverare"», in Gianni Celati. Traduzione, tradizione, riscrittura, a cura di M. Ronchi Stefanati, Roma, Aracne, p. 165-178.

Palmieri N., 2016a, «Cronologia», in Celati 2016b, p. LXIII-CXXIV.

Palmieri N., 2016b, «Notizie sui testi», in Celati 2016b, p. 1723-1788.

Pasolini P.P., [1967] 2004, «I segni viventi e i poeti morti», in Id., Empirismo eretico, in Saggi sulla letteratura e sull'arte, a cura di W. Siti e S. De Laude, Milano, Mondadori, p. 1573-1581.

Raccis G., 2018, “Dei giri in bicicletta tra un capitolo e l'altro”: cronotopi del Lunario», Elephant \& Castle, 19, Lunario del Paradiso, a cura di N. Palmieri, www.cav.unibg.it.

Rondini A., 2013, Gianni Celati e la teoria letteraria del vento volatore, Macerata, Eum.

Schwarz-Lausten P., 2002, «L'abbandono del soggetto. Un'analisi del soggetto narrato e quello narrante nell'opera di Gianni Celati», Revue Romane, 1, p. 105-132.

Jousse Th., [1989] 1997, John Cassavetes, Torino, Lindau.

West R., 2000, Gianni Celati. The Craft of Everyday Storytelling, Toronto, University of Toronto Press.

\section{Filmografia}

Duck Soup, L. McCarey, USA, 1933.

Edge of the City, M. Ritt, USA, 1957.

Shadows, J. Cassavetes, USA, 1959.

Too Late Blues, J. Cassavetes, USA, 1961.

A Child Is Waiting, J. Cassavetes, USA, 1963.

Faces, J. Cassavetes, USA, 1968.

Easy Rider, D. Hopper, USA, 1969.

Husbands, J. Cassavetes, USA, 1970.

I Clowns, F. Fellini, Italia/Francia/Rft, 1970.

A Woman Under the Influence, J. Cassavetes, USA, 1974. 